\title{
Tipo de terapêutica e fatores de prognóstico na paralisia de Bell: estudo retrospectivo de cinco anos em um hospital português
}

\author{
Therapeutic approach and prognostic factors in Bell's palsy: \\ retrospective study of five years in a Portuguese hospital
}

\author{
Lília Andreia Mendes Ferraria ${ }^{\natural} \bowtie$, Maria Inês de Almeida Pinto da Silva ${ }^{2}$, Maria Helena Carneirinho Rosa ${ }^{1}$, \\ Luis Alberto Carvalho Jerónimo Antunes ${ }^{1}$ \\ ${ }^{1}$ Hospital Garcia de Orta, Almada, Portugal. \\ ${ }^{2}$ Centro Hospitalar Lisboa Central, Lisboa, Portugal.
}

\section{RESUMO}

Objetivos: Comparar as abordagens terapêuticas na paralisia de Bell (corticoterapia versus corticoterapia mais terapia antiviral, assim como tratamento fisioterápico) e investigar potenciais fatores relacionados com sua evolução clínica e prognóstico.

Métodos: Estudo retrospectivo dos casos de paralisia de Bell atendidos no Hospital Garcia de Orta, em Almada, Portugal, entre 2009 e 2013 (cinco anos). Avaliou-se a gravidade da disfunção inicial da paralisia pela escala de House-Brackmann. A avaliação das abordagens terapêuticas e dos fatores de prognóstico foi realizada com base na taxa e tempo de recuperação, sendo a recuperação considerada total quando o grau de House-Brackmann foi I após a terapêutica. Diabetes mellitus e gravidez e/ou puerpério foram avaliados também como fatores de risco para recorrência. Foram utilizados testes não paramétricos, com nível de significância $p<0,05$.

Resultados: Registraram-se 180 casos de paralisia de Bell no período do estudo, correspondendo a uma incidência média de 36 casos/ano. A principal terapêutica utilizada foi a combinação entre corticosteroide e antiviral, em $67,2 \%$ dos casos. A taxa de recuperação total com uso dessa combinação foi de $65,5 \%$ (57 de 87 doentes) versus 72,4\% (21 de 29 pacientes) com corticoterapia isolada. Relativamente ao tempo de recuperação, 72,4\% (63 de 87 doentes) recuperaram em três meses com o uso da combinação versus 75,8\% (22 de 29 doentes) com corticoterapia isolada. Não se verificou, portanto, associação estatisticamente significativa entre o tipo de terapêutica tanto relativamente à taxa $(\mathrm{p}=0,689)$ como relativamente ao tempo de recuperação $(\mathrm{p}=0,977)$. A presença de otalgia e/ou disgeusia foi o único fator estatisticamente relacionado com a evolução clínica, associando-se a uma menor taxa $(51,2 \%$ versus $74,4 \%)(p=0,004)$ e a um maior tempo de recuperação $(60,5 \%$ versus $79,2 \%$ de taxa de recuperação aos três meses) $(\mathrm{p}=0,011)$. Verificou-se associação estatisticamente significativa do tratamento fisioterápico com maior taxa de recuperação $(\mathrm{p}=0,049)$. Em $24,1 \%$ (sete de 29) dos doentes com diabetes mellitus houve recorrência da paralisia, contrastando com apenas 6,0\% (nove de 51) de recorrência em doentes sem diabetes mellitus ( $\mathrm{p}=0,006$ ).

Conclusões: Não houve diferença significativa na taxa de recuperação ou no tempo de recuperação da paralisia de Bell entre os casos que receberam terapêutica com corticosteroide e antiviral ou corticoterapia isolada. $\mathrm{O}$ tratamento fisioterápico associou-se à maior taxa de recuperação. Dos fatores de prognóstico estudados, apenas a queixa de otalgia e/ou disgeusia foi significativa, constituindo fator de mau prognóstico na paralisia de Bell. A presença de diabetes mellitus revelou-se fator de risco para recorrência da paralisia.

DESCRITORES: paralisia de Bell; corticosteroides; antivirais; prognóstico.

\section{ABSTRACT}

Aims: To compare therapeutic approaches (corticosteroids $v s$. corticosteroids + antivirals, as well as physical therapy) in patients with Bell's palsy and to investigate potential factors related to its clinical course and prognosis.

Methods: Retrospective study of clinical cases of Bell's palsy treated at Hospital Garcia de Orta, Almada, Portugal, between 2009 and 2013 (five years). The severity of the initial dysfunction caused by the palsy was assessed using the House-Brackmann scale. Therapeutic approaches and prognostic factors were evaluated based on the rate and time of recovery, and full recovery occurred when a House-Brackmann grade I was observed after therapy. Diabetes mellitus and pregnancy and/or the postpartum period were also evaluated as risk factors for recurrence. Non-parametric tests were used and the significance level was set as $\mathrm{p}<0.05$.

Results: One hundred and eighty cases of Bell's palsy were recorded throughout the study period, corresponding to an average incidence of 36 cases/year. The main therapeutic approach consisted of the combination of corticosteroids and antivirals in $67.2 \%$ of the cases. The full recovery rate with the combination therapy was $65.5 \%$ (57 out of 87 patients) vs. $72.4 \%$ (21 out of 29 patients) in corticosteroid therapy alone. As to recovery time, $72.4 \%$ (63 out of 87 patients) recovered in 3 months with the use of the combination therapy vs. $75.8 \%$ ( 22 out of 29 patients) in those treated with corticosteroids alone. Therefore, no statistical significance between the type of therapy used and recovery rate $(\mathrm{p}=0.689)$ or recovery time $(\mathrm{p}=0.977)$. Only otalgia and/or dysgeusia was/were statistically associated with the clinical course, showing a lower recovery rate $(51.2 \%$ vs. $74.4 \%)(\mathrm{p}=0.004)$ and longer recovery time $(60.5 \%$ vs. $79.2 \%$ at 3 months $)(p=0.011)$. There was a statistically significant association of physical therapy with higher recovery rate $(\mathrm{p}=0.049)$. Palsy recurred in $24.1 \%$ of the patients with diabetes $(7$ out of 29) compared with only $6.0 \%$ ( 9 out of 51) among non-diabetic patients ( $\mathrm{p}=0.006$ ).

Conclusions: No significant difference was observed in recovery rate or recovery time among the cases treated with the combined therapy and those treated with corticosteroids alone. Physical therapy was associated with a higher recovery rate. Among the prognostic factors, only otalgia and/or dysgeusia was/were statistically significant, indicating a poor prognosis of Bell's palsy. The presence of diabetes mellitus proved to be a risk factor for recurrence.

KEY WORDS: Bell palsy; adrenal cortex hormones; antiviral agents; prognosis. 


\section{INTRODUÇÃO}

Sir Charles Bell, no século XIX, foi o primeiro a descrever a anatomia e a função do nervo facial, dando origem à designação de paralisia de Bell, definida por uma disfunção idiopática do VII par craniano, de instalação aguda, resultando em paralisia completa ou parcial da mímica facial [1]. A paralisia pode ser precedida por otalgia intensa e associar-se a distúrbios do paladar (disgeusia), salivação e lacrimejamento, além de hiperacusia. Os primeiros sinais clínicos são mais frequentemente detectados ao acordar [2]. A paralisia de Bell constitui a causa mais frequente de paralisia facial, com uma incidência estimada entre 20 e 30 casos por 100 mil habitantes, com prevalência ligeiramente superior no sexo feminino. É rara antes dos 10 anos de idade e a sua incidência é bimodal, com picos na terceira e oitava décadas de vida. Como fatores de risco para a paralisia de Bell têm sido relatados hipertensão arterial, diabetes mellitus, gravidez, puerpério e infecção pelo vírus herpes tipo 1. Frequentemente autolimitada, pode causar temporariamente dificuldade na mastigação e no fechamento da pálpebra do lado afetado, o que pode resultar em lesão de córnea pelo ressecamento do olho [3].

Na maioria dos casos o curso natural da paralisia de Bell é favorável. No entanto, aproximadamente $30 \%$ dos doentes sofrem algumas sequelas e $4 \%$ terão sequelas graves, como sincinésias (movimentos involuntários que acompanham movimentos desejados) [4]. A terapêutica instituida tem como finalidade melhorar a função da mímica facial e facilitar a recuperação, prevenindo as sequelas. Múltiplos artigos têm sido publicados sobre a terapêutica e fatores de prognóstico da paralisia de Bell, contudo esses aspectos ainda se mantêm controversos.

Este estudo teve como objetivo caracterizar, epidemiológica e clinicamente, a população de doentes com paralisia de Bell atendidos no serviço de urgência de otorrinolaringologia do Hospital Garcia de Orta, localizado em Almada, Portugal. Pretende-se comparar as abordagens terapêuticas na paralisia de Bell (corticoterapia isolada versus corticosteroide e antiviral) quanto à taxa e ao tempo de recuperação, levando em conta a gravidade da disfunção inicial. Pretendeu-se ainda identificar potenciais fatores de prognóstico e de recorrência da paralisia de Bell.

\section{MÉTODOS}

Foi realizada uma revisão retrospectiva de todos os prontuários clínicos de pacientes admitidos no serviço de urgência de otorrinolaringologia durante o período de janeiro de 2009 a dezembro de 2013 (cinco anos), com o diagnóstico de paralisia de Bell. Foram incluídos todos os pacientes com história compatível com paralisia facial periférica de origem idiopática. O estudo teve aprovação da Comissão de Ética do Hospital Garcia de Orta E. P. E. (no 07/2015).

Foi feita uma caraterização epidemiológica com base na idade, sexo e sazonalidade. Clinicamente, foram avaliadas as seguintes variáveis: antecedentes pessoais; hemiface afetada; sintomatologia concomitante; gravidade da paralisia segundo a escala de House-Brackmann [5], que considera os casos de maior gravidade os que apresentam uma disfunção inicial de grau $\geq$ VI e normal um grau igual a I (Quadro 1); abordagem terapêutica utilizada (de

Quadro 1. Avaliação do grau de paralisia facial segundo escala de House-Brackmann [5].

\begin{tabular}{|c|c|c|c|c|}
\hline \multirow{2}{*}{ Grau } & \multirow{2}{*}{ Descrição } & \multicolumn{3}{|c|}{ Características } \\
\hline & & Geral & No repouso & Ao movimento \\
\hline 1 & Normal & \multicolumn{3}{|l|}{ Função facial normal em todas as áreas } \\
\hline II & Disfunção leve & $\begin{array}{l}\text { Leve fraqueza notável apenas à } \\
\text { inspeção próxima; pode haver } \\
\text { sincinesia muito discreta. }\end{array}$ & $\begin{array}{l}\text { Simetria e tônus } \\
\text { normais }\end{array}$ & $\begin{array}{l}\text { TESTA: função boa a moderada } \\
\text { OLHO: fechamento completo com mínimo esforço } \\
\text { BOCA: leve assimetria }\end{array}$ \\
\hline III & Disfunção moderada & $\begin{array}{l}\text { Diferença óbvia, mas não } \\
\text { desfigurante, entre os dois lados; } \\
\text { sincinesia e/ou espasmo hemifacial } \\
\text { notáveis, mas não severos }\end{array}$ & $\begin{array}{l}\text { Simetria e tônus } \\
\text { normais }\end{array}$ & $\begin{array}{l}\text { TESTA: movimento moderado a leve } \\
\text { OLHO: fechamento completo com esforço } \\
\text { BOCA: levemente fraca com o máximo esforço }\end{array}$ \\
\hline IV & $\begin{array}{l}\text { Disfunção moderadamente } \\
\text { severa }\end{array}$ & $\begin{array}{l}\text { Fraqueza óbvia e/ou } \\
\text { assimetria desfigurante }\end{array}$ & $\begin{array}{l}\text { Simetria e tônus } \\
\text { normais }\end{array}$ & $\begin{array}{l}\text { TESTA: nenhum movimento } \\
\text { OLHO: fechamento incompleto } \\
\text { BOCA: assimetria com o máximo esforço }\end{array}$ \\
\hline V & Disfunção severa & $\begin{array}{l}\text { Apenas uma movimentação } \\
\text { discretamente perceptível }\end{array}$ & Assimetria & $\begin{array}{l}\text { TESTA: nenhum movimento } \\
\text { OLHO: fechamento incompleto } \\
\text { BOCA: movimento discreto }\end{array}$ \\
\hline $\mathrm{VI}$ & Paralisia total & Nenhum movimento & & \\
\hline
\end{tabular}


acordo com o critério clínico do médico assistente): corticoterapia isolada, antiviral isolado, corticoterapia e antiviral, tratamento fisioterápico; tempo de início do tratamento em relação com o início dos sintomas; tempo de recuperação; presença de recuperação, sendo a recuperação considerada total quando o grau de House-Brackmann era igual a I após a terapêutica. A avaliação do grau de paralisia facial segundo a escala de House-Brackmann é feita por rotina em todos os casos, como prática do serviço de Otorrinolaringologia. Não houve nenhum critério de exclusão relativamente ao tempo de início do tratamento medicamentoso ou fisioterápico.

Consideraram-se os seguintes fatores de prognóstico relativamente à taxa e tempo de recuperação: sexo, gravidade da paralisia, recidiva dos episódios, presença ou não de diabetes mellitus e hipertensão arterial, queixa de otalgia e/ou disgeusia e tipo de terapêutica utilizada. Gravidez e/ou puerpério e presença de diabetes mellitus foram avaliados como possíveis fatores de recorrência da doença.

Os dados quantitativos foram descritos através da média e desvio padrão, e os dados categóricos por frequências e percentagens. Realizaram-se análises bivariadas entre os fatores de prognóstico e o tempo e taxa de recuperação e entre os fatores de recorrência e a recorrência da paralisia de Bell, utilizando testes não paramétricos - teste exato de Fisher e qui quadrado de Pearson - com um nível de significância de $\mathrm{p}<0,05$. A análise estatística foi realizada no programa IBM SPSS versão 20.0.

\section{RESULTADOS}

Registraram-se 180 casos de paralisia de Bell nos cinco anos entre 2009 e 2013 no Hospital Garcia de Orta, o que corresponde a uma incidência média de 36 casos/ano. A média de idade dos pacientes foi de 48,719,6 anos (variação de 11 a 91 anos) e houve um discreto predomínio de pacientes do sexo feminino (56,7\%). A incidência máxima ocorreu entre os 21 e os 40 anos (33,9\% dos casos), encontrando-se uma menor incidência nos dois extremos de idade (Tabela 1).

Os antecedentes pessoais mais frequentemente encontrados foram distúrbios cardiovasculares, com predomínio da hipertensão arterial sistêmica $(31,1 \%)$, seguidos de diabetes mellitus (16\%) e dislipidemia (15\%), sendo que $2,8 \%$ dos pacientes da amostra eram mulheres grávidas ou no puerpério (Tabela 1).

Clinicamente, a maioria dos pacientes apresentava disfunção inicial de gravidade moderada a severa $(51,0 \%)$, não se verificando predomínio da hemiface
Tabela 1. Caracterização epidemiológica e clínica da população do estudo, constituída por 180 pacientes, observados no serviço de urgência de Otorrinolaringologia do Hospital Garcia de Orta, em Almada, Portugal, no período 2009-2013.

\begin{tabular}{|c|c|c|}
\hline Características & $\mathbf{n}^{*}$ & $\%$ \\
\hline $\begin{array}{l}\text { Sexo }(n=180) \\
\quad \text { Feminino }\end{array}$ & 102 & 56,7 \\
\hline $\begin{array}{l}\text { Idade }(\mathrm{n}=180) \\
\begin{array}{l}0-20 \text { anos } \\
\text { 21-40 anos } \\
\text { 41-60 anos } \\
61-80 \text { anos } \\
81-100 \text { anos }\end{array}\end{array}$ & $\begin{array}{l}11 \\
61 \\
52 \\
48 \\
8\end{array}$ & $\begin{array}{c}6,1 \\
33,9 \\
28,9 \\
26,7 \\
4,4\end{array}$ \\
\hline $\begin{array}{l}\text { Sazonalidade }(\mathrm{n}=180) \\
\text { Primavera } \\
\text { Verão } \\
\text { Outono } \\
\text { Inverno }\end{array}$ & $\begin{array}{l}43 \\
36 \\
41 \\
60\end{array}$ & $\begin{array}{c}23,9 \\
20 \\
22,7 \\
33,3\end{array}$ \\
\hline $\begin{array}{l}\text { Hemiface afetada }(n=180) \\
\text { Esquerda }\end{array}$ & 91 & 50,6 \\
\hline $\begin{array}{l}\text { Período do dia dos } 1^{0_{S}} \text { sinais de paralisia }(n=180) \\
\text { Ao acordar } \\
\text { Outros }\end{array}$ & $\begin{array}{c}42 \\
138\end{array}$ & $\begin{array}{l}23,3 \\
76,7\end{array}$ \\
\hline $\begin{array}{l}\text { Gravidade da paralisia segundo escala de } \\
\text { House-Brackmann }(\mathrm{n}=159)+ \\
\text { Disfunção ligeira - Grau II } \\
\text { Disfunção moderada - Grau III } \\
\text { Disfunção moderada a severa - Grau IV } \\
\text { Disfunção severa - Grau V }\end{array}$ & $\begin{array}{l}17 \\
46 \\
81 \\
15\end{array}$ & $\begin{array}{c}10,7 \\
28,9 \\
51,0 \\
9,4\end{array}$ \\
\hline $\begin{array}{l}\text { Antecedentes pessoais }(\mathrm{n}=180) \\
\text { Sem antecedentes } \\
\text { Doenças cardiovasculares } \\
\text { Doenças auto-imunes } \\
\text { Doenças oncológicas } \\
\text { Doenças atópicas } \\
\text { Doenças infetocontagiosas }\end{array}$ & $\begin{array}{c}90 \\
112 \\
8 \\
4 \\
3 \\
2\end{array}$ & $\begin{array}{c}41 \\
51 \\
4 \\
2 \\
1 \\
1\end{array}$ \\
\hline $\begin{array}{l}\text { Sintomatologia acompanhante }(n=166)+ \\
\text { Sem sintomatologia acompanhante } \\
\text { Otalgia + disgeusia } \\
\text { Disgeusia } \\
\text { Otalgia }\end{array}$ & $\begin{array}{l}116 \\
22 \\
17 \\
11\end{array}$ & $\begin{array}{c}69,9 \\
13,3 \\
10,2 \\
6,6\end{array}$ \\
\hline $\begin{array}{l}\text { Recorrência da paralisia }(n=180) \\
\quad \text { Sim }\end{array}$ & 16 & 8,9 \\
\hline $\begin{array}{l}\text { Abordagem terapêutica farmacológica }(\mathrm{n}=177)+ \\
\text { Corticoterapia + valaciclovir } \\
\text { Corticoterapia + aciclovir } \\
\text { Corticoterapia } \\
\text { Valaciclovir } \\
\text { Sem terapêutica }\end{array}$ & $\begin{array}{c}111 \\
8 \\
48 \\
3 \\
7\end{array}$ & $\begin{array}{c}62,7 \\
4,5 \\
27,1 \\
1,7 \\
4,0\end{array}$ \\
\hline $\begin{array}{l}\text { Abordagem terapêutica - fisiatria }(n=156)+ \\
\text { Com acompanhamento }\end{array}$ & 120 & 76,9 \\
\hline $\begin{array}{l}\text { Taxa de recuperação }(n=177)+ \\
\text { Recuperação total } \\
\text { Recuperação parcial } \\
\text { Não } \\
\text { Desconhecida }\end{array}$ & $\begin{array}{c}83 \\
39 \\
3 \\
52\end{array}$ & $\begin{array}{c}46,9 \\
22,0 \\
1,7 \\
29,4\end{array}$ \\
\hline $\begin{array}{l}\text { Tempo de recuperação }(n=125)+ \\
\leq 15 \text { dias } \\
\text { 15-30dias } \\
\text { 30-90 dias } \\
\text { 90-180 dias } \\
\geq 180 \text { dias } \\
\text { Sem recuperação }\end{array}$ & $\begin{array}{c}4 \\
47 \\
40 \\
13 \\
18 \\
3\end{array}$ & $\begin{array}{c}3,2 \\
37,6 \\
32,0 \\
10,4 \\
14,4 \\
2,4\end{array}$ \\
\hline
\end{tabular}

* Vários pacientes apresentavam mais do que um antecedente pessoal. † $\mathrm{n}$ diferente de 180 por ausência de dados em alguns prontuários. 
afetada. A recorrência da paralisia facial foi registada em $8,9 \%$ dos pacientes, sendo que esta afetou mais frequentemente a hemiface contralateral à afetada primariamente (Tabela 1).

Foi apenas possível avaliar a abordagem terapêutica em 177 pacientes, pois em três deles não constava o tipo de terapêutica no prontuário. A principal abordagem terapêutica utilizada foi a associação entre corticosteroide e antiviral, em $119(67,2 \%)$ casos, enquanto 48 pacientes $(27,1 \%)$ receberam apenas corticoterapia. O tempo médio de início do tratamento em relação ao início dos sintomas foi de 1,27 dias, não havendo diferença entre o tempo de início do tratamento nas diferentes abordagens terapêuticas utilizadas. A maioria dos pacientes (76,9\%) foi orientada para tratamento fisioterápico com um tempo médio de início de 6,38 dias em relação ao início dos sintomas. Houve recuperação em $68,9 \%$ dos pacientes (total em 46,9\%, parcial em 22,0\%). A recuperação foi mais frequentemente atingida entre os 15-30 dias após o inicio do quadro, mas em 180 dias a taxa de recuperação atingiu os $83,2 \%$. Essa taxa foi independente do grau de disfunção inicial da paralisia (Tabela 1).

Para avaliar os fatores de prognóstico foram somados os casos em que havia otalgia e/ou disgeusia. Apenas a presença de otalgia e/ou disgeusia esteve estatisticamente associada à evolução clínica, com menor taxa e maior tempo de recuperação aos três meses, constituindo um fator de mau prognóstico. Não se verificou, nesta amostra, associação entre a presença de diabetes mellitus, hipertensão, dislipidemia e gravidez e o nível de recuperação da doença (Tabelas 2 e 3).

Relativamente à terapêutica, não se encontrou diferença estatisticamente significativa entre a combinação de corticoterapia e antiviral relativamente à corticoterapia isolada, no que diz respeito à taxa de recuperação. Verificou-se uma associação estatisticamente significativa entre acompanhamento em tratamento fisioterápico e maior taxa de recuperação o que não aconteceu relativamente ao tempo de recuperação (Tabelas 4 e 5).

Tabela 2. Avaliação dos fatores prognósticos quanto à taxa de recuperação da paralisia de Bell, na amostra de 125 pacientes em que foi possível obter informações sobre a recuperação, observados no Serviço de Urgência de Otorrinolaringologia do Hospital Garcia de Orta. Almada, Portugal, 2009-2013.

\begin{tabular}{|c|c|c|c|c|}
\hline \multirow[b]{2}{*}{ Fator prognóstico } & \multicolumn{4}{|c|}{ Taxa de recuperação } \\
\hline & $\begin{array}{l}\text { Recuperação total } \\
\text { n (\%) }\end{array}$ & $\begin{array}{c}\text { Recuperação parcial } \\
\text { n (\%) }\end{array}$ & $\begin{array}{c}\text { Sem recuperação } \\
\text { n (\%) }\end{array}$ & $\mathbf{p}^{*}$ \\
\hline \multicolumn{5}{|l|}{ Sexo } \\
\hline Feminino & $47(67,1)$ & $22(31,4)$ & $1(1,5)$ & \multirow{2}{*}{0,726} \\
\hline Masculino & $36(65,5)$ & $17(30,9)$ & $2(3,6)$ & \\
\hline \multicolumn{5}{|l|}{ Presença de diabetes mellitus } \\
\hline Sim & $16(72,7)$ & $6(27,3)$ & $0(0,0)$ & \multirow{2}{*}{0,483} \\
\hline Não & $67(65,0)$ & $33(32,0)$ & $3(3,0)$ & \\
\hline \multicolumn{5}{|c|}{ Presença de hipertensão arterial } \\
\hline Sim & $32(72,7)$ & $12(27,3)$ & $0(0,0)$ & \multirow{2}{*}{0,187} \\
\hline Não & $51(63,0)$ & $27(33,3)$ & $3(3,7)$ & \\
\hline \multicolumn{5}{|l|}{ Gravidez e puerpério } \\
\hline Sim & $1(33,3)$ & $2(66,7)$ & $0(0,0)$ & \multirow{2}{*}{0,427} \\
\hline Não & $82(67,2)$ & $37(30,3)$ & $3(2,5)$ & \\
\hline \multicolumn{5}{|c|}{ Presença de otalgia e/ou disgeusia } \\
\hline $\operatorname{Sim}$ & $22(51,2)$ & $21(48,8)$ & $0(0,0)$ & \multirow{2}{*}{0,004} \\
\hline Não & $61(74,4)$ & $18(22,0)$ & $3(3,6)$ & \\
\hline \multicolumn{5}{|l|}{$\begin{array}{l}\text { Gravidade segundo escala de } \\
\text { House-Brackmann }\end{array}$} \\
\hline ligeira a moderada & $34(72,3)$ & $11(23,4)$ & $2(4,3)$ & \multirow{2}{*}{0,227} \\
\hline moderada a severa & $49(62,8)$ & $28(35,9)$ & $1(1,3)$ & \\
\hline \multicolumn{5}{|l|}{ Recidiva da paralisia facial } \\
\hline Sim & $6(50,0)$ & $6(50,0)$ & $0(0,0)$ & \multirow{2}{*}{0,289} \\
\hline Não & $77(68,1)$ & $33(29,2)$ & $3(2,7)$ & \\
\hline
\end{tabular}

* Teste do qui quadrado. 
Tabela 3. Avaliação dos fatores prognósticos quanto ao tempo de recuperação da paralisia de Bell, na amostra de 125 pacientes nos quais foi possível obter informações sobre a recuperação, observados no Serviço de Urgência de Otorrinolaringologia do Hospital Garcia de Orta. Almada, Portugal, 2009-2013.

\begin{tabular}{|c|c|c|c|c|c|c|c|}
\hline \multirow{2}{*}{ Fator prognóstico } & \multicolumn{7}{|c|}{ Tempo de recuperação } \\
\hline & $\begin{array}{c}\leq 15 \text { dias } \\
\mathbf{n}(\%)\end{array}$ & $\begin{array}{c}\text { 15-30 dias } \\
\text { n (\%) }\end{array}$ & $\begin{array}{l}\text { 30-90 dias } \\
\text { n }(\%)\end{array}$ & $\begin{array}{c}90-180 \text { dias } \\
\text { n (\%) }\end{array}$ & $\begin{array}{l}\geq 180 \text { dias } \\
\quad \mathrm{n}(\%)\end{array}$ & $\begin{array}{c}\text { Sem recuperação } \\
n(\%)\end{array}$ & $\mathbf{p}^{*}$ \\
\hline \multicolumn{8}{|l|}{ Sexo } \\
\hline Feminino & $4(5,7)$ & $26(37,2)$ & $19(27,1)$ & $7(10,0)$ & $13(18,6)$ & $1(1,4)$ & \multirow{2}{*}{0,123} \\
\hline Masculino & $0(0)$ & $21(38,2)$ & $21(38,2)$ & $6(10,9)$ & $5(9,1)$ & $2(3,6)$ & \\
\hline \multicolumn{8}{|l|}{ Presença de diabetes mellitus } \\
\hline Sim & $0(0)$ & $10(45,5)$ & $5(22,7)$ & $3(13,6)$ & $4(18,2)$ & $0(0)$ & \multirow{2}{*}{0,492} \\
\hline Não & $4(3,9)$ & $37(35,9)$ & $35(34)$ & $10(9,7)$ & $14(13,6)$ & $3(2,9)$ & \\
\hline \multicolumn{8}{|c|}{ Presença de hipertensão arterial } \\
\hline Sim & $1(2,3)$ & $15(34,1)$ & $17(38,6)$ & $5(11,4)$ & $6(13,6)$ & $0(0)$ & \multirow{2}{*}{0,547} \\
\hline Não & $3(3,7)$ & $32(39,5)$ & $23(28,4)$ & $8(9,9)$ & $12(14,8)$ & $3(3,7)$ & \\
\hline \multicolumn{8}{|l|}{ Gravidez e puerpério } \\
\hline Sim & $0(0)$ & $1(33,3)$ & $1(33,3)$ & $1(33,3)$ & $0(0)$ & $0(0)$ & \multirow{2}{*}{0,817} \\
\hline Não & $4(3,3)$ & $46(37,6)$ & $39(32)$ & $12(9,8)$ & $18(14,8)$ & $3(2,5)$ & \\
\hline \multicolumn{8}{|c|}{ Presença de otalgia e/ou disgeusia } \\
\hline Sim & $0(0)$ & $12(27,9)$ & $14(32,6)$ & $9(20,9)$ & $8(18,6)$ & $0(0)$ & \multirow{2}{*}{0,011} \\
\hline Não & $4(4,9)$ & $35(42,6)$ & $26(31,7)$ & $4(4,9)$ & $10(12,2)$ & $3(3,7)$ & \\
\hline \multicolumn{8}{|l|}{$\begin{array}{l}\text { Gravidade segundo escala de } \\
\text { House-Brackmann }\end{array}$} \\
\hline Ligeira a moderada & $3(6,4)$ & $19(40,4)$ & $15(31,8)$ & $6(12,8)$ & $2(4,3)$ & $2(4,3)$ & \multirow{2}{*}{0,066} \\
\hline Moderada a severa & $1(1,3)$ & $28(35,8)$ & $25(32,1)$ & $7(9)$ & $16(20,5)$ & $1(1,3)$ & \\
\hline \multicolumn{8}{|l|}{ Recidiva da paralisia facial } \\
\hline Sim & $0(0)$ & $2(16,7)$ & $5(41,6)$ & $2(16,7)$ & $3(25)$ & $0(0)$ & \multirow{2}{*}{0,418} \\
\hline Não & $4(3,5)$ & $45(39,8)$ & $35(31)$ & $11(9,7)$ & $15(13,3)$ & $3(2,7)$ & \\
\hline
\end{tabular}

* Teste do qui quadrado.

Tabela 4. Análise bivariada entre a taxa de recuperação da paralisia de Bell e a abordagem terapêutica, na amostra de pacientes em que foi possível obter informações sobre a recuperação. Terapêutica farmacológica: $n=116$. Fisioterapia: $n=125$. Serviço de urgência de Otorrinolaringologia do Hospital Garcia de Orta. Almada, Portugal, 2009-2013.

\begin{tabular}{|c|c|c|c|c|}
\hline \multirow{2}{*}{ Abordagem terapêutica } & \multicolumn{4}{|c|}{ Taxa de recuperação } \\
\hline & $\begin{array}{c}\text { Recuperação total } \\
\text { n (\%) }\end{array}$ & $\begin{array}{c}\text { Recuperação parcial } \\
\text { n (\%) }\end{array}$ & $\begin{array}{c}\text { Sem recuperação } \\
\text { n (\%) }\end{array}$ & $\mathbf{p}^{*}$ \\
\hline Corticoterapia isolada & $21(72,4)$ & $7(24,1)$ & $1(3,4)$ & \multirow{2}{*}{0,689} \\
\hline Corticoterapia + antiviral & $57(65,5)$ & $28(32,2)$ & $2(2,3)$ & \\
\hline Com fisioterapia & $71(65,7)$ & $36(33,3)$ & $1(0,9)$ & \multirow{2}{*}{0,049} \\
\hline Sem fisioterapia & $12(70,6)$ & $3(17,6)$ & $2(11,8)$ & \\
\hline
\end{tabular}

* Teste do qui quadrado.

Tabela 5. Análise bivariada entre o tempo de recuperação da paralisia de Bell e a abordagem terapêutica, na amostra de pacientes em que foi possível obter informações sobre a recuperação. Terapêutica farmacológica: $n=116$. Fisioterapia: $n=125$. Serviço de urgência de Otorrinolaringologia do Hospital Garcia de Orta. Almada, Portugal, 2009-2013.

\begin{tabular}{|c|c|c|c|c|c|c|c|}
\hline \multirow[b]{2}{*}{ Abordagem terapêutica } & \multicolumn{7}{|c|}{ Tempo de recuperação } \\
\hline & $\begin{array}{c}\leq 15 \text { dias } \\
n(\%)\end{array}$ & $\begin{array}{c}15-30 \text { dias } \\
n(\%)\end{array}$ & $\begin{array}{c}30-90 \text { dias } \\
\text { n ( } \%)\end{array}$ & $\begin{array}{c}90-180 \text { dias } \\
n(\%)\end{array}$ & $\begin{array}{c}\geq 180 \text { dias } \\
n(\%)\end{array}$ & $\begin{array}{c}\text { Sem recuperação } \\
\text { n (\%) }\end{array}$ & $\mathbf{p}^{*}$ \\
\hline Corticoterapia isolada & $1(3,4)$ & $10(34,5)$ & $11(37,9)$ & $2(6,9)$ & $4(13,8)$ & $1(3,4)$ & \multirow{2}{*}{0,977} \\
\hline Corticoterapia + antiviral & $2(2,3)$ & $33(37,9)$ & $28(32,2)$ & $9(10,3)$ & $13(14,9)$ & $2(2,3)$ & \\
\hline Fisioterapia & $3(2,8)$ & $40(37,0)$ & $36(33,3)$ & $12(11,1)$ & $16(14,8)$ & $1(0,9)$ & \multirow{2}{*}{0,315} \\
\hline Sem fisioterapia & $1(5,9)$ & $7(41,2)$ & $4(23,5)$ & $1(5,9)$ & $2(11,8)$ & $2(11,8)$ & \\
\hline
\end{tabular}

* Teste do qui quadrado. 
Tabela 6. Análise bivariada entre a taxa de recuperação da paralisia de Bell e a abordagem terapêutica, numa amostra de 73 pacientes com grau de disfunção inicial $\geq$ IV de House-Brackmann, observados no serviço de urgência de Otorrinolaringologia do Hospital Garcia de Orta. Almada, Portugal, 2009-2013.

\begin{tabular}{|c|c|c|c|c|}
\hline \multirow{2}{*}{ Abordagem terapêutica } & \multicolumn{4}{|c|}{$\begin{array}{c}\text { Taxa de recuperação } \\
\text { ( } n=73 \text { com grau de disfunção inicial } \geq I V \text { de House-Brackmann) }\end{array}$} \\
\hline & $\begin{array}{l}\text { Recuperação total } \\
\text { n (\%) }\end{array}$ & $\begin{array}{l}\text { Recuperação parcial } \\
\text { n (\%) }\end{array}$ & $\begin{array}{l}\text { Sem recuperação } \\
\text { n (\%) }\end{array}$ & $\mathbf{p}^{*}$ \\
\hline Corticoterapia isolada & $13(68,4)$ & $6(31,6)$ & $0(0)$ & \multirow{2}{*}{0,697} \\
\hline Corticoterapia+antiviral & $34(63,0)$ & $19(35,2)$ & $1(1,8)$ & \\
\hline
\end{tabular}

* Teste do qui quadrado.

Tabela 7. Análise bivariada entre o tempo de recuperação da paralisia de Bell e a abordagem terapêutica, numa amostra de 73 pacientes com grau de disfunção inicial $\geq$ IV de House-Brackmann, observados no serviço de urgência de Otorrinolaringologia do Hospital Garcia de Orta. Almada, Portugal, 2009-2013.

\begin{tabular}{|c|c|c|c|c|c|c|c|}
\hline \multirow[b]{2}{*}{ Terapêutica farmacológica } & \multicolumn{7}{|c|}{ Tempo de recuperação } \\
\hline & $\begin{array}{c}\leq 15 \text { dias } \\
n(\%)\end{array}$ & $\begin{array}{l}\text { 15-30 dias } \\
\text { n (\%) }\end{array}$ & $\begin{array}{c}30-90 \text { dias } \\
\text { n }(\%)\end{array}$ & $\begin{array}{c}\text { 90-180 dias } \\
\text { n (\%) }\end{array}$ & $\begin{array}{c}\geq 180 \text { dias } \\
\text { n (\%) }\end{array}$ & $\begin{array}{c}\text { Sem recuperação } \\
n(\%)\end{array}$ & $\mathbf{p}^{*}$ \\
\hline Corticoterapia isolada & $0(0)$ & $6(31,6)$ & $8(42,1)$ & $1(5,3)$ & $4(21,0)$ & $0(0)$ & \multirow{2}{*}{0,848} \\
\hline Corticoterapia + antiviral & $1(1,8)$ & $19(35,2)$ & $17(31,5)$ & $5(9,3)$ & $11(20,4)$ & $1(1,8)$ & \\
\hline
\end{tabular}

* Teste do qui quadrado.

Nos casos de maior gravidade (96 casos dos quais se excluíram 23 por ausência de dados) com disfunção inicial da paralisia de grau $\geq$ IV de House-Brackmann também não houve diferença na taxa $(p=0,697)$ nem no tempo de recuperação $(p=0,848)$ (Tabelas 6 e 7).

Dos fatores de risco estudados para recorrência da paralisia de Bell (gravidez, puerpério e diabetes mellitus), apenas a presença de diabetes mellitus revelou-se fator de risco com significância estatística: em $24,1 \%$ dos pacientes com diabetes mellitus houve recorrência da paralisia, contrastando com recorrência da paralisia em apenas $6,0 \%$ dos doentes sem diabetes $(p=0,006)$ (Tabela 8).

Tabela 8. Análise bivariada entre a diabetes mellitus, gravidez /puerpério e a recorrência da paralisia facial, numa amostra de 180 pacientes, observados no serviço de urgência de Otorrinolaringologia do Hospital Garcia de Orta. Almada, Portugal, 2009-2013.

\begin{tabular}{lccc}
\hline \multicolumn{1}{c}{ Fatores de risco } & \multicolumn{3}{c}{ Recorrência } \\
\cline { 2 - 4 } & $\begin{array}{c}\text { Sem recorrência } \\
\mathbf{n}(\%)\end{array}$ & $\begin{array}{c}\text { Com recorrência } \\
\mathbf{n}(\%)\end{array}$ & $\mathbf{p}^{*}$ \\
Com diabetes mellitus & $22(75,9)$ & $7(24,1)$ & $\mathbf{0 , 0 0 6}$ \\
Sem diabetes mellitus & $142(94,0)$ & $9(6,0)$ & \\
Grávida/puérpera & $4(80,0)$ & $1(20,0)$ & 0,376 \\
Sem gravidez/puerpério & $160(91,4)$ & $15(8,6)$ & \\
\hline
\end{tabular}

* Teste do qui quadrado.

\section{DISCUSSÃO}

Sob o ponto de vista epidemiológico, nesta amostra a incidência de paralisia de Bell foi máxima entre os 21 e os 40 anos. O mesmo verificou-se no estudo de Yanagihara [6], em que a incidência máxima ocorreu entre a segunda e a quarta décadas de vida. Sazonalmente, Spengos et al. [7] obtiveram resultados semelhantes aos do presente estudo, com um declínio da paralisia de Bell no verão e um pico de incidência no inverno.

Tal como neste estudo, Kanoh et al. [8] verificaram que a maioria das pessoas com paralisia de Bell detectam os primeiros sinais da doença pela manhã, ao acordar, apresentando no seu estudo uma relação de 191:30:36 (manhã:tarde:noite). Isto pode ser explicado pelo fato do nervo facial sofrer um processo de isquemia que, mais frequentemente, aumenta e progride lentamente durante a noite, dando origem a edema e compressão do nervo e consequente perda da sua função.

Verificou-se neste estudo que $68,9 \%$ dos pacientes recuperaram a mímica facial. Este resultado mostra-se concordante com o estudo de Peitersen [9], em que $71 \%$ dos pacientes recuperaram a função normal da mímica facial.

$\mathrm{Na}$ literatura, o principal fator prognóstico descrito é o grau da paralisia facial, apresentando pior prognóstico os doentes com paralisia facial igual ou superior ao grau IV na escala de House-Brackmann. 
Outros fatores, como hiperacusia, diminuição da lágrima, idade superior a 60 anos, diabetes mellitus, hipertensão arterial sistêmica, ausência do reflexo estapédico (também conhecido por reflexo acústico, consistindo em contração dos músculos do ouvido médio em resposta a um estímulo sonoro) e presença de dor intensa, são também indicadores de mau prognóstico [10]. Nesta amostra, apenas a presença de otalgia e/ou disgeusia esteve estatisticamente relacionada com a taxa e o tempo de recuperação, associando-se a menor taxa e a maior tempo para a recuperação, constituindo-se, portanto, em fator de mau prognóstico na paralisia de Bell.

Quanto à recorrência da paralisia facial, o dado encontrado nesta casuística $(8,9 \%$ de recorrência) concorda com o estudo de Reixach-Casulá et al. [11], que descreveram uma taxa de recorrência de $7 \%$ a $15 \%$. O único fator de risco com significância estatística para a recorrência da paralisia facial encontrado no presente estudo foi a presença de diabetes mellitus.

A escolha da abordagem terapêutica para a paralisia de Bell continua a ser um problema comum na prática médica [12-17]. Os resultados deste estudo relativamente à terapêutica antiviral estão de acordo com a revisão Cochrane de 2015 [18], que incluiu 11 ensaios controlados, com um total de 2883 pacientes com paralisia de Bell. A revisão concluiu não haver benefício na combinação de corticosteroides com antivirais, comparados aos corticosteroides isolados ou associados a placebo, assim como nenhum benefício foi encontrado no uso de antivirais isoladamente quando comparados a placebo [17].

Antunes et al. [19] analisaram o efeito de valaciclovir (1500 mg/dia) em 46 indivíduos com paralisia de Bell, num ensaio randomizado controlado, e não encontraram beneficio estatisticamente significativo na associação terapêutica relativamente à taxa de recuperação.

No que diz respeito ao tratamento fisioterapêutico, a metanálise de Pereira et al. [20] concluiu que a fisioterapia melhora a funcionalidade facial nos pacientes com paralisia de Bell, promovendo a simetria da face, controlando e reduzindo as sincinésias. $\mathrm{O}$ mesmo se verificou no presente estudo, em que o acompanhamento fisioterapêutico associou-se significativamente ao aumento da taxa de recuperação.

Pode-se concluir que dos potenciais fatores de prognóstico estudados nesta amostra, apenas a presença de otalgia e/ou disgeusia constituiu fator de mau prognóstico na paralisia de Bell, tendo-se associado à diminuição da taxa de recuperação e aumento do tempo para a recuperação. Por sua vez o diabetes mellitus revelou-se um fator de risco para recorrência da paralisia. A combinação entre corticosteroide e antiviral não apresentou diferença na taxa ou no tempo de recuperação relativamente à corticoterapia isolada, independentemente da gravidade da disfunção inicial da paralisia de Bell. O acompanhamento com fisioterapia associou-se à maior taxa de recuperação.

\section{NOTA}

Declaração de conflitos de interesse

Os autores declaram não haver conflitos de iteresse relevantes ao conteúdo deste estudo.

\section{REFERÊNCIAS}

1. Gilden DH. Clinical practice. Bell's Palsy. N Engl J Med. 2004 Sept 23;351(13):1323-31. http://dx.doi.org/10.1056/NEJMcp041120

2. Kanoh N, Nomura J, Satomi F. Nocturnal onset and development of Bell's palsy. Laryngoscope. 2005 Jan;115(1):99-100. http://dx.doi. org/10.1097/01.mlg.0000150700.46377.96

3. Valença MM, Valença LP, Lima MC. Paralisia facial periférica idiopática de Bell: a propósito de 180 doentes. Arq Neuropsiquiatr. 2001 Sept;59(3-B):733-9. http://dx.doi.org/10.1590/S0004-282X2001000500016

4. Peitersen E. Bell's palsy: the spontaneous course of 2,500 peripheral facial nerve palsies of different etiologies. Acta Otolaryngol Suppl. 2002;(549):4-30. http://dx.doi.org/10.1080/000164802760370736

5. House JW, Brackmann DE. Facial nerve grading system. Otolaryngol Head Neck Surg. 1985 Apr;93(2):146-7.

6. Yanagihara N. Incidence of Bell's palsy. Ann Otol Rhinol Laryngol Suppl. 1988 Nov-Dec;137:3-4.

7. Spengos K, Sameli S, Stouraitis G, Kolias A, Koulouri O, Kokkinos Z, Makrylou I, Tsivgoulis A, Tsivgoulis G, Vassilopoulos D. Seasonal variation of Bell's palsy in Athens, Greece - a hospital-based retrospective evaluation over fifteen years. Eur Neurol. 2006;55(2):84-8. http://dx.doi.org/10.1159/000092779

8. Kanoh N, Nomura J, Satomi F. Nocturnal onset and development of Bell's palsy. Laryngoscope. 2005 Jan;115(1):99-100. http://dx.doi. org/10.1097/01.mlg.0000150700.46377.96 
9. Peitersen E. The natural history of Bell's palsy. Am J Otol. 1982 Oct;4(2):107-11.

10. Douglas E. Clinical disorders of the facial nerve. In: Flint PW, Haughey BH, Lund VJ, Niparko JK, Richardson MA, Robbins KT, Thomas JR, editors. Cummings otolaryngology: head and neck surgery. 5th ed. Philadelphia: Mosby Elsevier; 2010. p. $2391-401$.

11. Reixach-Casulá R, Beramendi PG, Sanchez EP. Paralisia facial periférica recidivante e alternante: registo de um caso com 5 episódios. Arq Neuropsiquiatr. 1982 Dec;40(4):382 http://dx.doi.org/10.1590/S0004-282X1982000400009

12. Goudakos JK, Markou KD. Corticosteroids vs corticosteroids plus antiviral agents in the treatment of Bell palsy: a systematic review and meta-analysis. Arch Otolaryngol Head Neck Surg. 2009 June;135(6):558-64. http://dx.doi.org/10.1001/archoto.2009.44

13. Kawaguchi K, Inamura H, Abe Y, Koshu H, Takashita E, Muraki Y, Matsuzaki Y, Nishimura H, Ishikawa H, Fukao A, Hongo S, Aoyagi M.Reactivation of herpes simplex vírus type 1 and varicella-zoster vírus and therapeutic effects of combination therapy with prednisolone and valacyclovir in patients with Bell's palsy. Laryngoscope. 2007 Jan;117(1):147-56. http://dx.doi.org/10.1097/01.mlg.0000248737.65607.9e

14. Lagalla G, Logullo F, Di Bella P, Provinciali L, Ceravolo MG. Influence of early high-dose steroid treatment on Bell's palsy evolution. Neurol Sci. 2002 Sept;23(3):107-12. http://dx.doi.org/10.1007/s100720200035

15. De Diego JI, Prim MP, De Sarriá MJ, Madero R, Gavilán J. Idiopathic facial paralysis: a randomized, prospective, and controlled study using single-dose prednisone versus acyclovir three times daily. Laryngoscope. 1998 Apr;108(4 Pt 1):573-5. http://dx.doi.org/10.1097/00005537199804000-00020

16. Hato N, Matsumoto S, Kisaki H, Takahashi H, Wakisaka H, Honda N, Gyo K, Murakami S, Yanagihara N. Efficacy of early treatment of Bell's palsy with oral acyclovir and prednisolone. Otol Neurotol. 2003 Nov;24(6):948-51. http://dx.doi.org/10.1097/00129492-20031100000022

17. Axelsson S, Berg T, Jonsson L, Engström M, Kanerva M, Stjernquist-Desatnik A. Bell's palsy - the effect of prednisolone and/or valaciclovir versus placebo in relation to baseline severity in a randomised controlled trial. Clin Otolaryngol. 2012 Aug;37(4):283-90. http://dx.doi. org/10.1111/j.1749-4486.2012.02526.x

18. Gagyor I, Madhok VB, Daly F, Somasundara D, Sullivan M, Gammie F, Sullivan F. WITHDRAWN. Antiviral treatment for Bell's palsy (idiopathic facial paralysis). Cochrane Database Syst Rev. 2015 May 4;5:CD001869.

19. Antunes ML, Fukuda Y, Testa JRG. Clinical treatment of Bell's palsy: comparative study among valaciclovir plus deflazacort, deflazacort and placebo. Acta AWHO 2000;19:68-75.

20. Pereira LM, Obara K, Dias JM, Menacho MO, Lavado EL, Cardoso JR. Facial exercise therapy for facial palsy: systematic review and meta-analysis. Clin Rehabil. 2011 July;25(7):649-58. http://dx.doi.org/10.1177/0269215510395634 